\title{
Comparação entre Três Métodos de Obtenção do Índice de Respiração Rápida e Superficial em Pacientes Submetidos ao Desmame da Ventilação Mecânica*
}

\section{Comparison among Three Methods to Measure the Rapid Shallow Breathing Index in Patients Submitted to Weaning from Mechanical Ventilation}

Lorena de Oliveira Santos ${ }^{1}$, Maraísa Rodrigues Borges', Luciana Castilho de Figueirêdo², Cristina Aparecido Veloso Guedes ${ }^{3}$, Bruna Scharlack Vian ${ }^{4}$, Karina Kappaz ${ }^{5}$, Sebastião Araújo6.

\section{RESUMO}

JUSTIFICATIVA E OBJETIVOS: Esta pesquisa justificase na necessidade de maior conhecimento a respeito das técnicas de desmame ventilatório utilizadas em pacientes sob ventilação mecânica prolongada. O objetivo deste estudo foi comparar a obtenção do índice de respiração rápida e superficial (IRRS) nas modalidades PSV de $10 \mathrm{cmH}_{2} \mathrm{O}$ e PEEP de $5 \mathrm{cmH}_{2} \mathrm{O}$ (PSV10); CPAP de $5 \mathrm{cmH}_{2} \mathrm{O}$ (CPAP5) e em ventilação espontânea (ESP), correlacionando com sucesso ou insucesso na retirada da ventilação mecânica (VM).

MÉTODO: Estudo prospectivo incluindo 54 pacientes em VM há mais de 48 horas, submetidos ao IRRS em três

1. Fisioterapeuta Especialista em Fisioterapia Respiratória na UTI Adulto da UNICAMP.

2. Fisioterapeuta Titular do Programa de Aprimoramento Profissional da FUNDAP em Fisioterapia em UTI Adulto da FCM-UNICAMP.

3. Mestre em Cirurgia, Área de Pesquisa Experimental pela FCM/ UNICAMP, Supervisora dos Cursos de Especialização e Aprimoramento em Fisioterapia Respiratória em UTI Adulto.

4. Fisioterapeuta da UTI Adulto da UNICAMP

5. Fisioterapeuta Plantonista do Hospital AC Camargo e Nossa Senhora de Lourdes

6. Coordenador da Disciplina de Fisiologia e Metabologia Cirúrgica do Departamento de Cirurgia da FCM-UNICAMP

*Recebido do Hospital de Clínicas da Faculdade de Ciências Médicas da Universidade Estadual de Campinas (HC-FCM-UNICAMP), Campinas, SP.

Apresentado em 27 de março de 2007

Aceito para publicação em 17 de julho de 2007

Endereço para correspondência:

Lorena de Oliveira Santos

Travessa Curuzú, 1458

66093-540 Belém, PA

Fones: (91) 3246-5678 - 8153-1771

E-mail: lorenasantos@gmail.com

(C)Associação de Medicina Intensiva Brasileira, 2007 modos ventilatórios: PSV10, CPAP5 e ESP nos momentos pré e pós-nebulização, utilizando tubo-T. Os pacientes foram retirados da VM quando o IRRS era $<105$.

RESULTADOS: Não houve diferença estatisticamente significativa entre os valores do IRRS obtidos nos momentos pré e pós-nebulização. Houve diferença estatística significativa dos valores do IRRS entre CPAP5 e PSV10 $(p=0,008)$ e entre a modalidade ESP e PSV10 $(p=0,01)$ no momento pré-nebulização e dos valores do IRRS obtidos entre CPAP5 e PSV10 $(p=0,01)$ no momento pós-nebulização.

CONCLUSÕES: Neste estudo pode-se observar que o valor do IRRS foi superestimado quando obtido na modalidade PSV10. Foi constatado também que não houve necessidade de nebulização de 30 minutos antes da extubação traqueal quando o desmame é realizado com a técnica de redução gradativa da PSV. Este estudo sugeriu que o IRRS foi capaz de prever o sucesso do desmame; entretanto, incapaz de determinar o insucesso quando seu valor fosse $<105$. Recomenda-se que o IRRS seja analisado em associação com outros parâmetros preditivos de desmame.

Unitermos: desmame, índice de respiração rápida e superficial, ventilação com pressão de suporte, ventilação mecânica, tubo-T

\section{SUMMARY}

BACKGROUND AND OBJECTIVES: To compare the attainment of the rapid shallow breathing index (IRRS) in modalities PSV $10 \mathrm{cmH}_{2} \mathrm{O}$ and PEEP $5 \mathrm{cmH}_{2} \mathrm{O}$ (PSV10), CPAP $5 \mathrm{cmH}_{2} \mathrm{O}$ (CPAP5) and spontaneous breathing $(\mathrm{SB})$, correlating them with success on failure in the withdrawal of mechanical ventilation (MV).

METHODS: Prospective study including 54 patients in MV > 48 hours, submitted to the IRRS in three ventila- 
tory modalities: PSV10, CPAP5 and SB at the moments before and after T-tube spontaneous breathing. The patients were removed from MV when IRRS was < 105.

RESULTS: There wasn't statistically significant difference between IRRS values at the moments before and after T-tube SB. There was statistically significant difference IRRS value between the modalities CPAP5 and PSV10 $(p=0.008)$, and between the modalities SB and PSV10 $(p=0.01)$ at the moment before T-Tube SB and of IRRS value, gotten between CPAP5 and PSV10 $(p=0.01)$ at the moment after T-tube SB.

CONCLUSIONS: From this sample, it can be observed that IRRS values are overestimated when gotten in modality PSV10. It was also evidenced that there is no need of a 30 min T-tube SB before extubation, when the weaning is performed with the technique of gradual reduction of PSV. This study suggested that IRRS is able to predict weaning success; however it is not able to determine failure when it was $<105$. It is recommended that IRRS must be analyzed in association with other predictive weaning parameters.

Key Words: mechanical ventilation, pressure support ventilation, rapid shallow breathing index, T-tube, weaning

\section{INTRODUÇÃO}

Aproximadamente $90 \%$ dos pacientes críticos necessitam de ventilação mecânica (VM) e o processo da retirada do suporte ventilatório representa $40 \%$ do tempo total do seu uso'.

Os pacientes com dificuldades na retirada da VM necessitam maior tempo de internação, aumentando os riscos de morbidade e mortalidade com maior incidência de pneumonia associada à VM, atelectasias, traqueobronquite necrosante, bem como o aumento dos custos hospitalares ${ }^{2-5}$. No entanto, a extubação precoce pode resultar em re-intubação, fadiga ou instabilidade cardiovascular'.

Alguns índices preditivos são utilizados à beira do leito para predizer a capacidade do paciente em manter adequada ventilação espontânea. Dos diversos índices preditivos sugeridos, o índice de respiração rápida e superficial (IRRS) parece ser o parâmetro mais útil por sua simplicidade e confiabilidade ${ }^{3}$.

O IRRS é mensurado através da relação entre a freqüência respiratória (FR) e o volume-corrente $(\mathrm{Vt})$ obtido pela ventilometria. O procedimento de medida é realizado a partir da obtenção do volume-minuto (Ve), enquanto o paciente ventila espontaneamente. Valores superiores a 105 indicam insucesso na retirada do suporte ventilatório ${ }^{6}$.

Estudos mostraram que o IRRS é superior aos parâme- tros convencionais em predizer o resultado da retirada da VM, devido a maior exatidão nos resultados ${ }^{3}$.

Lee e col. ${ }^{7}$ avaliaram o IRRS com pacientes conectados ao ventilador nos modos pressão positiva contínua nas vias aéreas (CPAP) ou ventilação com pressão de suporte (PSV), mostrando prejuízo na acurácia preditiva, porém sem descrever os níveis de suporte pressórico utilizados.

Rodrigues e col. ${ }^{8}$ mostraram que, em unidade de terapia intensiva (UTI) da cidade de São Paulo, o modo PSV, com níveis variando entre 6 e $12 \mathrm{cmH}_{2} \mathrm{O}$, é extensivamente utilizado durante a avaliação dos parâmetros de retirada da VM e extubação.

El-Khatib e col. ${ }^{9}$ mostraram que houve diminuição de $49 \%$ no valor do IRRS quando a avaliação foi realizada com o paciente conectado ao ventilador mecânico no modo CPAP.

O objetivo deste estudo foi comparar a obtenção do IRRS nas modalidades PSV de $10 \mathrm{cmH}_{2} \mathrm{O}$ e pressão expiratória positiva final (PEEP) de $5 \mathrm{cmH}_{2} \mathrm{O}$ (PSV10); CPAP de $5 \mathrm{cmH}_{2} \mathrm{O}$ (CPAP5) e em ventilação espontânea (ESP), correlacionando com o sucesso e o insucesso na retirada da VM.

\section{MÉTODO}

Trata-se de um estudo prospectivo composto por uma população de 54 pacientes internados na UTI e Unidade Coronariana (UCO) do Hospital das Clínicas da Universidade Estadual de Campinas (UNICAMP). O protocolo do estudo foi previamente aprovado pelo Comitê de Ética e Pesquisa da Instituição sob o parecer 287/2006.

Os critérios de inclusão foram idade $>16$ anos; pacientes sob VM há mais de 48 horas; pacientes utilizando respiradores da marca Bird ${ }^{\circledR} 8400$ ou Hamilton Medical RAPHAEL; fração inspirada de oxigênio $\left(\mathrm{FiO}_{2}\right) \leq 40 \%$; PEEP de $5 \mathrm{cmH}_{2} \mathrm{O}$; índice de oxigenação > 200; pacientes sem sedação contínua ou intermitente; pacientes com estabilidade hemodinâmica e hidroeletrolítica; sem fármacos vasoativos ou utilizando dopamina até $5 \mu \mathrm{g} /$ $\mathrm{kg} / \mathrm{min}$ e/ou dobutamina até $10 \mu \mathrm{g} / \mathrm{kg} / \mathrm{min}$.

O IRRS foi obtido inicialmente com o paciente em VM na modalidade PSV10. Após, ainda em ventilação artificial, o índice foi obtido na modalidade CPAP5. Ambas as medidas utilizaram o Vt, Ve e FR através do display do respirador. A última medida foi obtida com o paciente ventilando espontaneamente, sem suporte ventilatório, utilizando-se o ventilômetro de Wright ${ }^{\circledR}$.

Os pacientes que apresentaram o IRRS superior a 105 permaneceram em VM e os pacientes que apresentaram o 
IRRS inferior a 105 foram submetidos à nebulização com tubo-T por 30 minutos e, após o procedimento, foi mensurado novamente o IRRS nos três modos ventilatórios, inicialmente com o ventilômetro de Wright ${ }^{\circledR}$, uma vez que o paciente já estava sem suporte ventilatório, seguido da VM com CPAP5 e logo após PSV10, respectivamente.

Os valores obtidos foram comparados e, permanecendo inferiores a 105, o paciente foi retirado da VM. Se ele apresentasse estabilidade clínica em até 24 horas era considerado como sucesso na retirada da prótese ventilatória, confirmando os valores de IRRS mensurados. O fluxograma do estudo encontra-se na figura 1.

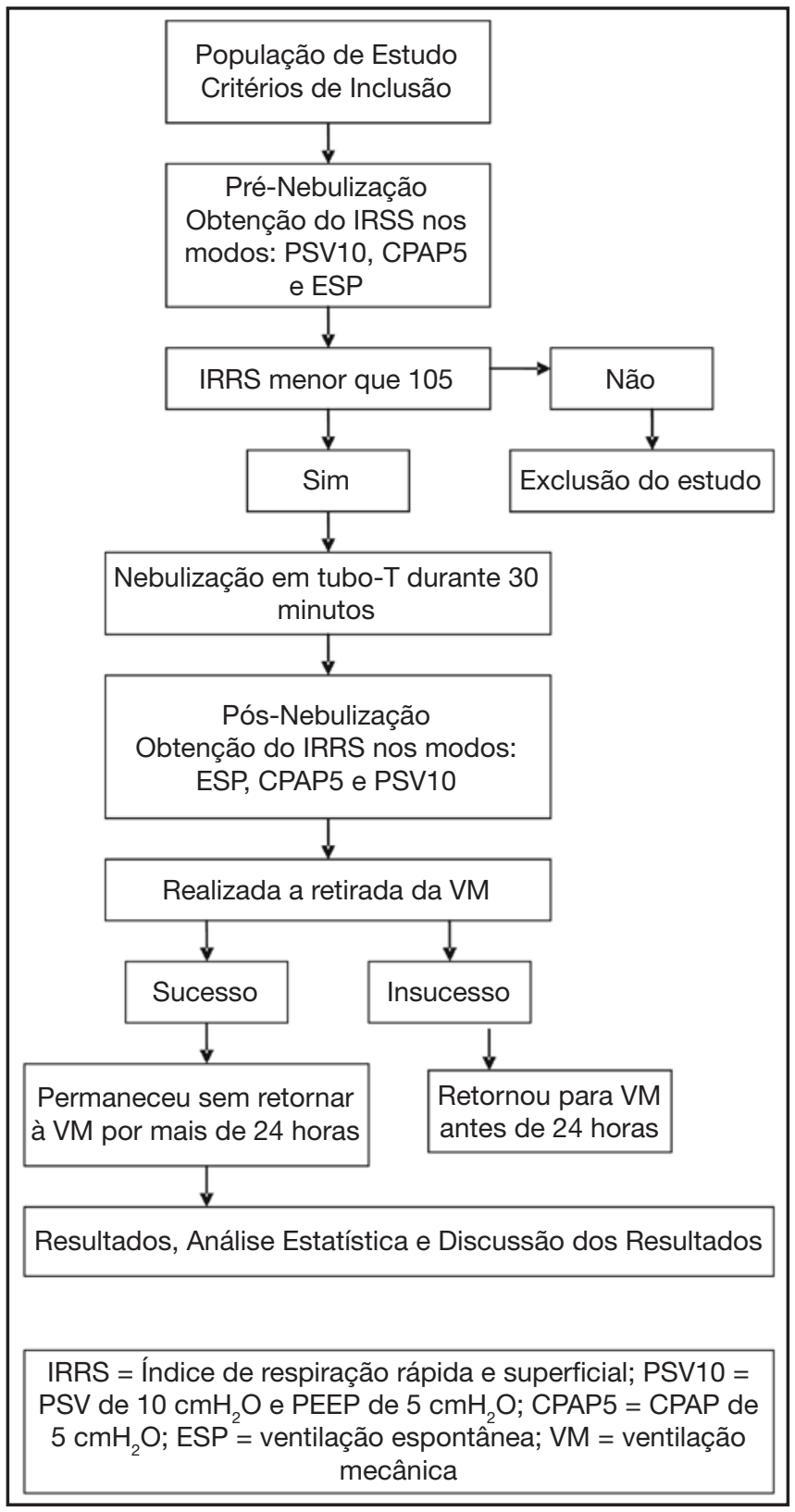

Figura 1 - Fluxograma do Estudo
Para a análise estatística foram utilizados os testes não paramétrico de Wilcoxon e de Kruskal-Wallis. O nível significativo adotado foi de $5 \%(p<0,05)$.

\section{RESULTADOS}

Os dados demográficos e os diagnósticos de internação na UTI e UCO encontram-se na tabela 1.

Tabela 1 - Dados Demográficos e Diagnósticos dos Pacientes Estudados $(n=54)$

\begin{tabular}{lc}
\hline Variáveis $^{*}$ & \\
\hline Idade (anos) & \\
Sexo & $47,35 \pm 18,19$ \\
$\quad$ Feminino & $18(31 \%)$ \\
$\quad$ Masculino & $36(69 \%)$ \\
Tempo de ventilação mecânica (dias) & $11,35 \pm 7,99$ \\
Diagnóstico & \\
Insuficiência respiratória aguda & $20 \%$ \\
$\quad$ Pós-operatório de cirurgia neurológica eletiva & $17 \%$ \\
$\quad$ Traumatismo cranioencefálico & $17 \%$ \\
$\quad$ Pós-operatório de cirurgia cardíaca & $15 \%$ \\
$\quad$ Acidente vascular encefálico hemorrágico & $11 \%$ \\
$\quad$ Quadro infeccioso & $7 \%$ \\
$\quad$ Outro & $13 \%$ \\
\hline
\end{tabular}

*Valores expressos em Média \pm DP

A tabela 2 contém os valores do IRRS obtidos nos momentos pré e pós-nebulização com tubo-T na população estudada. Não houve diferença estatisticamente significativa entre os valores do IRRS obtidos nas modalidades estudadas entre os momentos estabelecidos. Houve diferença estatisticamente significativa dos valores do IRRS entre a modalidade CPAP5 e PSV10, e entre a modalidade ESP e PSV10 no momento pré-nebulização. Também houve diferença estatisticamente significativa do valor do IRRS obtido na modalidade CPAP5 e PSV10 no momento pós-nebulização (Figura 2).

A tabela 3 ilustra os valores do IRRS obtidos nas modalidades estudadas, nos momentos pré e pós-nebulização, relacionados ao sucesso e insucesso no desmame da VM. Houve diferença estatisticamente significativa entre o IRRS obtido na modalidade CPAP5 e ESP em relação à PSV10 no momento pré-nebulização, para os pacientes que obtiveram insucesso no desmame da VM. Houve diferença estatisticamente significativa entre as modalidades CPAP5 e PSV10 no momento prénebulização e entre as modalidades CPAP5 e ESP em relação à PSV10 no momento pós-nebulização para os pacientes que obtiveram sucesso no desmame da VM (Figura 2). 
Tabela 2 - Distribuição dos Valores do Índice de Respiração Rápida e Superficial (IRRS) nas Modalidades CPAP5, ESP e PSV10 nos Momentos Pré e Pós-Nebulização.

\begin{tabular}{|c|c|c|c|c|c|c|c|}
\hline Momentos & Condição & Média \pm DP & Mínimo & $1^{\circ}$ Quartil & Mediana & $3^{\circ}$ Quartil & Máximo \\
\hline \multirow[t]{3}{*}{ Pré } & CPAP5 & $67,65 \pm 35,57$ & 18,00 & 45,75 & 62,00 & 83,25 & 242,00 \\
\hline & ESP & $59,02 \pm 22,46$ & 14,00 & 44,00 & 58,00 & 68,43 & 123,00 \\
\hline & PSV10 & $48,61 \pm 19,46$ & 11,00 & 37,00 & 47,50 & 58,00 & 115,00 \\
\hline \multirow[t]{3}{*}{ Pós } & CPAP5 & $71,32 \pm 47,84$ & 12,00 & 45,50 & 61,50 & 85,20 & 327,00 \\
\hline & ESP & $64,31 \pm 35,69$ & 14,00 & 44,75 & 56,50 & 79,25 & 226,00 \\
\hline & PSV10 & $55,23 \pm 31,51$ & 9,00 & 35,45 & 48,80 & 65,25 & 198,00 \\
\hline
\end{tabular}

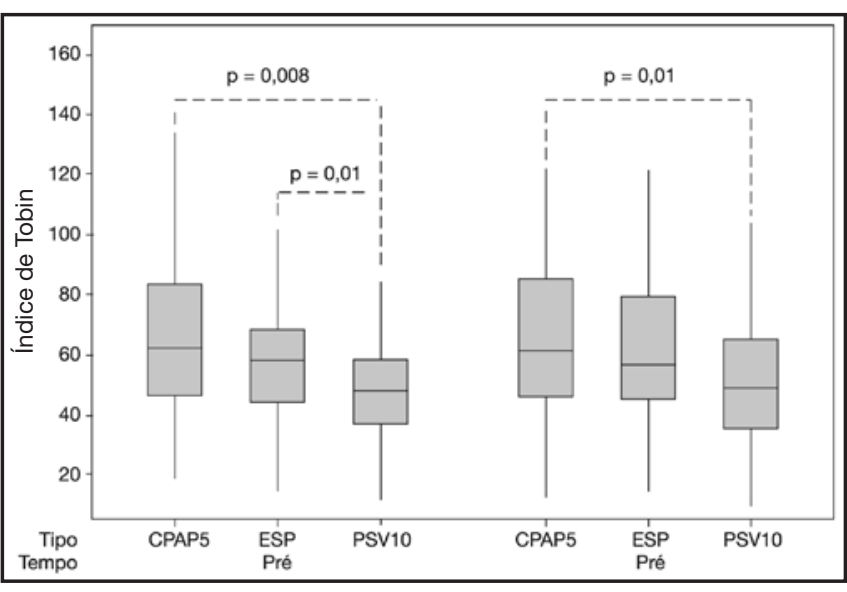

Figura 2 - Comparação entre os Valores do Índice de Respiração Rápida e Superficial (IRRS) Obtidos nas Modalidades CPAP5, ESP e PSV10 nos Momentos Pré e Pós-Nebulização com Tubo-T.

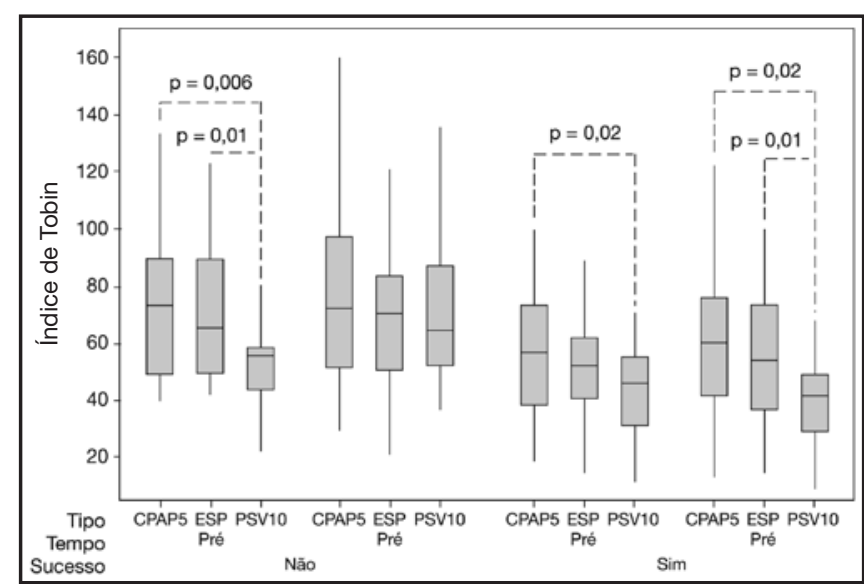

Figura 3 - Comparação entre os Valores do Índice de Respiração Rápida e Superficial (IRRS) Obtidos nas Modalidades CPAP5, ESP e PSV10 entre os Momentos Pré Pós-Nebulização em Relação aos Pacientes que Apresentaram Sucesso e Insucesso na Retirada da VM.

Tabela 3 - Índice de Respiração Rápida e Superficial (IRRS) nas Modalidades CPAP5, ESP e PSV10 nos Momentos Pré e Pós-Nebulização com Sucesso ou Insucesso no Desmame da VM.

\begin{tabular}{|c|c|c|c|c|c|c|c|c|c|}
\hline Sucesso & $\mathrm{n}$ & Momento & Condição & Média \pm DP & Mínimo & $1^{\circ}$ Quartil & Mediana & $3^{\circ}$ Quartil & Máximo \\
\hline \multirow[t]{6}{*}{ Não } & 23 & Pré & CPAP5 & $79,40 \pm 42,96$ & 39,00 & 49,00 & 73,00 & 90,00 & 242,00 \\
\hline & & & ESP & $69,28 \pm 22,07$ & 41,00 & 49,00 & 65,00 & 89,00 & 123,00 \\
\hline & & & PSV10 & $52,55 \pm 18,08$ & 13,00 & 43,50 & 56,00 & 58,60 & 84,20 \\
\hline & & Pós & CPAP5 & $85,70 \pm 60,10$ & 29,00 & 51,00 & 72,00 & 97,00 & 327,00 \\
\hline & & & ESP & $75,96 \pm 43,00$ & 20,00 & 50,00 & 70,00 & 83,00 & 226,00 \\
\hline & & & PSV10 & $73,52 \pm 36,27$ & 36,00 & 51,60 & 64,00 & 87,00 & 198,00 \\
\hline \multirow[t]{6}{*}{ Sim } & 31 & Pré & CPAP5 & $58,93 \pm 26,42$ & 18,00 & 38,00 & 57,00 & 73,00 & 140,00 \\
\hline & & & ESP & $51,41 \pm 19,84$ & 14,00 & 40,00 & 52,00 & 62,00 & 106,00 \\
\hline & & & PSV10 & $45,68 \pm 20,21$ & 11,00 & 31,40 & 45,60 & 55,00 & 115,00 \\
\hline & & Pós & CPAP5 & $60,64 \pm 33,46$ & 12,00 & 41,20 & 60,00 & 76,00 & 172,00 \\
\hline & & & ESP & $55,66 \pm 26,71$ & 14,00 & 36,60 & 54,00 & 73,00 & 136,00 \\
\hline & & & PSV10 & $41,66 \pm 18,52$ & 9,00 & 29,00 & 41,00 & 49,00 & 101,00 \\
\hline
\end{tabular}

$\mathrm{n}=$ número de pacientes

\section{DISCUSSÃO}

A intubação e a utilização da VM estão associadas a diversas complicações. No entanto, a retirada da VM e a extubação devem ser realizadas assim que o paciente estiver apto a sustentar ventilação espontânea adequada e troca gasosa eficaz ${ }^{10,11}$. Identificar o momento mais apropriado para a retirada da VM e predizer com precisão o sucesso da extubação tem sido um objetivo constante na UTI ${ }^{12,13}$.

Embora muitos pacientes apresentem condições estáveis para a retirada da VM, a ventilação espontânea muitas vezes não é sustentada, necessitando a sua re-instalação. Muitos fatores podem influenciar esse 
processo e dentre eles destacam-se os parâmetros ventilatórios; os critérios usados para definir sucesso ou insucesso; o momento em que o paciente está sendo estudado; diferentes protocolos clínicos da UTI e diferentes populações. Isto sugere a importância de se identificar os índices preditivos ${ }^{14}$.

Os índices preditivos são usados para prognosticar a capacidade do paciente em sustentar ventilação espontânea adequada. Alguns parâmetros baseados na mecânica respiratória, troca gasosa e padrão ventilatório têm sido propostos como índices preditivos úteis para orientar e definir o melhor momento para a retirada da $\mathrm{VM}^{2}$.

O IRRS, introduzido por Yang e Tobin ${ }^{6}$, demonstraram ser o mais efetivo entre todos os índices descritos na literatura para predizer o sucesso ou insucesso no desmame ${ }^{15-17}$. Os autores afirmam que valores do índice superiores a 105 indicam insucesso na retirada da VM.

No entanto, outros autores questionam a precisão desse índice ${ }^{7-9,14,18}$. Conti e col. ${ }^{14}$ realizaram estudo clínico e prospectivo analisando diversos índices preditivos, incluindo o IRRS, e concluíram que todos os índices são incapazes de predizer com precisão absoluta o momento ideal para a retirada da VM.

Soo Hoo e Park ${ }^{19}$ avaliando práticas de desmame em nove hospitais de Los Angeles, observaram variações na medida do modo ventilatório em que o IRRS foi obtido. Puderam concluir que a maioria ( $85 \%$ ) dos profissionais utilizou o modo CPAP para a medida, enquanto a minoria (15\%) utilizou o tubo-T.

Rodrigues e col. ${ }^{8}$ demonstraram que $91 \%$ dos fisioterapeutas que atuam em UTI no estado de São Paulo utilizam o modo PSV entre 6 e $12 \mathrm{cmH}_{2} \mathrm{O}$ para realizar a medida do IRRS durante a avaliação dos parâmetros da retirada do suporte ventilatório.

El Khatib e col. ${ }^{9}$ realizaram uma pesquisa com 32 pacientes em pós-operatório de cirurgia cardíaca, mensurando o IRRS em três condições: CPAP $5 \mathrm{cmH}_{2} \mathrm{O}$ com $\mathrm{FIO}_{2}$ de 0,4, CPAP $5 \mathrm{cmH}_{2} \mathrm{O}$ com $\mathrm{FIO}_{2}$ de 0,21 e ventilação espontânea durante um minuto. Seus resultados sugeriram que o IRRS foi influenciado pelo tipo de suporte ventilatório, com diminuição de $49 \%$ durante o modo CPAP, quando comparado à ventilação espontânea. Dessa forma, os autores concluíram que o IRRS pode ser aceitável se a determinação for realizada de forma similar aos aplicados por Yang e Tobin ${ }^{6}$, no qual os pacientes permanecem em ventilação espontânea por um minuto.

Em estudo realizado por Lee e col. ${ }^{7}$, com 52 pacientes, em que o IRRS foi medido nas modalidades CPAP e
PSV com o uso do display do ventilador, sendo que os pacientes foram extubados independentemente do valor do IRRS. Os autores observaram que não houve diferença estatisticamente significativa entre os valores de IRRS obtidos nas modalidades CPAP e PSV.

$\mathrm{O}$ estudo de Fiore e col. ${ }^{18}$ mostrou que a PSV, mesmo em níveis mínimos para compensar a resistência do tubo traqueal, gerou influência significativa nos valores do IRRS, diminuindo a FR, o Ve e o IRRS e aumentando o Vt. Recomendaram a aplicação rigorosa do método empregado nos estudos de Yang e Tobin ${ }^{6}$ para que não se prejudique a capacidade preditiva dos parâmetros. No presente estudo, os valores do IRRS em todas as modalidades mostraram-se inferiores a 105. No entanto, quando obtido no modo PSV10, o valor médio foi inferior quando comparado àqueles obtidos nas modalidades CPAP5 e ESP, indicando que a precisão do IRRS no modo PSV10 é superestimada. Estes achados sugerem que não é necessário o teste de nebulização com tubo-T por 30 minutos quando a evolução do desmame da VM for realizada com a redução gradativa da PSV até níveis mínimos $\left(10 \mathrm{cmH}_{2} \mathrm{O}\right)$. O tubo-T apresenta desvantagens, como o aumento do trabalho resistivo da ventilação pela presença do tubo traqueal e o colapso alveolar devido à perda da pressão positiva final residual ${ }^{20}$, além de levar à deterioração dos parâmetros de oxigenação durante o desmame, como sustentam Figueiredo e col. ${ }^{21}$.

O IRRS não avalia com precisão o sucesso do desmame, podendo ser empregado para obter uma tendência de sucesso ${ }^{22}$. Lee e col. ${ }^{7}$, em estudo prospectivo, concluíram que o IRRS maior que 105 não é incompatível com o sucesso do desmame ventilatório. Epstein e col. ${ }^{23}$ observaram também que houve sucesso na retirada da VM em pacientes que foram extubados com IRRS superior a 100. O autor atribuíu esse comportamento à presença de tubo traqueal de diâmetro pequeno ou lúmen reduzido por secreções, o que aumentaria o trabalho ventilatório. Também constatou insucesso na retirada da VM em pacientes que obtiveram IRRS menor que 100, sugerindo que ele pode ter menos precisão por não refletir a causa da falha no momento em que está sendo medido. Yang e Tobin ${ }^{6}$ demonstraram que o índice preditivo, quando aplicado individualmente, não prediz sucesso ou insucesso absoluto na extubação.

Diante do exposto, os resultados do presente estudo mostraram que o IRRS foi superestimado em sua precisão quando obtido na modalidade PSV10. Verificouse também que o teste de nebulização com tubo-T 
por 30 minutos não é necessário quando a evolução do desmame do paciente for realizada com a redução gradativa da PSV. O presente estudo ainda sugere que para a população estudada, o IRRS foi capaz de prever o sucesso do desmame da VM sem, contudo, ter sido capaz de determinar o insucesso na retirada do suporte ventilatório com valor menor que 105. Desta forma, recomenda-se que o IRRS seja analisado em associação com outros parâmetros preditivos de desmame para resultados com maior probabilidade de sucesso.

\section{REFERÊNCIAS}

01. Meade M, Guyatt G, Griffith L et al - Introduction to a series of systematic reviews of weaning from mechanical ventilation. Chest, 2001;120:(Suppl6):S396-S399.

02. Mancebo $\mathrm{J}$ - Weaning from mechanical ventilation. Eur Respir J, 1996;9:1923-1931.

03. Alia I, Esteban A - Weaning from mechanical ventilation. Crit Care, 2000;4:72-80.

04. Esteban A, Alia I, Gordo F et al - Extubation outcome after spontaneous breathing trials with T-tube or pressure support ventilation. Am J Respir Crit Care Med, 1997;156:459-465.

05. Assunção M, Machado F, Rosseti $\mathrm{H}$ et al - Avaliação de teste de tuboT como estratégia inicial de suspensão da ventilação mecânica. RBTI, 2006;18:121-125.

06. Yang KL, Tobin MJ - A prospective study of indexes predicting the outcome of trials of weaning from mechanical ventilation. $\mathrm{N}$ Engl J Med, 1991;324:1445-1450.

07. Lee KH, Hui KP, Chan TB et al - Rapid shallow breathing (frequency-tidal volume ratio) did not predict extubation outcome. Chest, 1994;105:540543.

08. Rodrigues MM, Fiore JF Jr, Benassule E et al - Variação na mensuração dos parâmetros da ventilação mecânica em hospitais da cidade de São Paulo. Rev Bras Fisioter, 2004;(Suppl):177.

09. El-Khatib MF, Jamaleddine GW, Khoury AR et al - Effect of continuous positive airway pressure on the rapid shallow breathing index in patients following cardiac surgery. Chest, 2002;121:475-479.

10. Marini JJ, Kelsen SG - Re-targeting ventilatory objectives in adult respiratory distress syndrome. New treatment prospects--persistent questions. Am Rev Respir Dis, 1992;146:2-3.

11. Parker JC, Hernandez LA, Peevy KJ - Mechanisms of ventilator-induced lung injury. Crit Care Med, 1993;21:131-143.

12. Petrini MF, Evans JN, Wall MA et al - Variability, reproducibility, and datacollection time of pulmonary bedside monitoring. Biomed Instrum Technol, 1998;32:273-281.

13. Biondi JW, Schulman DS, Soufer $R$ et al - The effect of incremental positive end-expiratory pressure on right ventricular hemodynamics and ejection fraction. Anesth Analg, 1998;67:144-151.

14. Conti G, Montini L, Pennisi MA et al - A prospective, blinded evaluation of indexes proposed to predict weaning from mechanical ventilation. Intensive Care Med, 2004;30:830-836.

15. Vassilakopoulos $\mathrm{T}$, Zakynthinos $\mathrm{S}$, Roussos $\mathrm{C}-$ The tension-time index and the frequency/tidal volume ratio are the major pathophysiologic determinants of weaning failure and success. Am J Respir Crit Care Med, 1998;158:378-385.

16. Krieger BP, Isber J, Breitenbucher A et al - Serial measurements of the rapid-shallow- breathing index as a predictor of weaning outcome in elderly medical patients. Chest, 1997;112:1029-1034

17. Borges VC, Andrade JRA, Lopes AC - Desmame da ventilação mecânica. Rev Bras Clin Terap, 1999;25:171-178.

18. Fiore JFJ, Oliveira ASCS. Pinho E et al - Uso de baixos níveis de pressão de suporte influencia a avaliação de parâmetros de desmame? RBTI, 2004;16:146-149.

19. Soo Hoo GW, Park L - Variation in the measurement of weaning parameters: a survey of respiratory therapists. Chest, 2002;121:1947-1955.

20. Brochard L, Rauss A, Benito S et al - Comparison of three methods of gradual withdrawal from ventilatory support during weaning from mechanical ventilation. Am J Respir Crit Care Med, 1994;150:896-903.

21. Figueiredo LC, Araujo S, kosour $\mathrm{C}$ et al - Comparação entre dois métodos de retirada do suporte ventilatório mecânico no pós-operatório de cirurgia cardíaca. RBTI, 2003;15:114-120.

22. Breitenbucher A, Ershowsky P, Krieger B - Rapid shallow breathing as a predictor of weaning outcome in the elderly. Am Rev Respir Dis, 1992;145:A520.

23. Epstein SK - Etiology of extubation failure and the predictive value of the rapid shallow breathing index. Am J Respir Crit Care Med, 1995;152:545549 . 